\title{
Abundance trends in kinematical groups of the Milky Way's disk ${ }^{\star}$
}

\author{
C. Soubiran and P. Girard
}

\begin{abstract}
Observatoire de Bordeaux, BP 89, 33270 Floirac, France
\end{abstract}
e-mail: soubiran@obs.u-bordeaux1.fr

Received 18 November 2004 / Accepted 19 March 2005

\begin{abstract}
We have compiled a large catalogue of metallicities and abundance ratios from the literature in order to investigate abundance trends of several alpha and iron peak elements in the thin disk and the thick disk of the Galaxy. The catalogue includes 743 stars with abundances of $\mathrm{Fe}, \mathrm{O}, \mathrm{Mg}, \mathrm{Ca}, \mathrm{Ti}, \mathrm{Si}, \mathrm{Na}$, Ni and $\mathrm{Al}$ in the metallicity range $-1.30<[\mathrm{Fe} / \mathrm{H}]<+0.50$. We have checked that systematic differences between abundances measured in the different studies were lower than random errors before combining them. Accurate distances and proper motions from Hipparcos and radial velocities from several sources have been retreived for 639 stars and their velocities $(U, V, W)$ and galactic orbits have been computed. Ages of 322 stars have been estimated with a Bayesian method of isochrone fitting. Two samples kinematically representative of the thin and thick disks have been selected, taking into account the Hercules stream which is intermediate in kinematics, but with a probable dynamical origin. Our results show that the two disks are chemically well separated, they overlap greatly in metallicity and both show parallel decreasing alpha elements with increasing metallicity, in the interval $-0.80<[\mathrm{Fe} / \mathrm{H}]<-0.30$. The Mg enhancement with respect to $\mathrm{Fe}$ of the thick disk is measured to be 0.14 dex. An even larger enhancement is observed for Al. The thick disk is clearly older than the thin disk with tentative evidence of an AMR over 2-3 Gyr and a hiatus in star formation before the formation of the thin disk. We do not observe a vertical gradient in the metallicity of the thick disk. The Hercules stream has properties similar to that of the thin disk, with a wider range of metallicity. Metal-rich stars assigned to the thick disk and super-metal-rich stars assigned to the thin disk appear as outliers in all their properties.
\end{abstract}

Key words. stars: abundances - stars: kinematics - Galaxy: disk - Galaxy: abundances -

Galaxy: kinematics and dynamics - catalogs

\section{Introduction}

It is now well accepted that the stellar disk of our Galaxy is made up of at least two components: the thin disk and the thick disk. The properties and origin of the thick disk are still matter of debate and require more investigation. In the solar neighbourhood, the observed distribution of stellar velocities is well approximated by the sum of two distinct velocity ellipsoids suggesting that the thin disk and the thick disk are discrete populations. In the last decade a number of studies have demonstrated that they also have distinct chemical distributions (Gratton et al. 1996; Fuhrmann 1998; Bensby et al. 2003; Mishenina et al. 2004, for instance). Unfortunatly, on the one hand, the statistics involving abundance ratios in the thick disk are rather poor, on the other hand, all these studies have not used the same criteria to define the thin disk and the thick disk populations. This paper is devoted to collecting the most chemical and kinematical data of high quality available in the literature and to investigate the chemical properties of the thin and thick disks identified by the simplest and most robust

* The catalogue of stellar parameters and abundances is only available in electronic form at the CDS via anonymous ftp to cdsarc.u-strasbg.fr $(130.79 .128 .5)$ or via http://cdsweb.u-strasbg.fr/cgi-bin/qcat?J/A+A/438/139 criterion, their velocity ellipsoids. Our aim is also to find the stellar parameters that allow the best separation of the thick disk and the thin disk for future investigations.

In order to study the properties of the thin disk and the thick disk separately, it is necessary to clearly identify the stars of each population. In practice, this is not obvious because their distributions overlap. The kinematical information is often used as a robust criterion for nearby stars but the deconvolution of velocity distributions is made complex by the fact that moving groups, superclusters and dynamical streams may exist and translate into inhomogeneities in the velocity ellipsoids. This problem has been recently revisited by Famaey et al. (2004) who identified several kinematical subgroups in a local sample of giants, representative of the solar neighbourhood. Among them, the Hercules stream is crucial because its motion, which is believed to have a dynamical origin, can be confused with that of the thick disk. Famaey et al. (2004) have estimated that $6.5 \%$ of their sample belongs this stream. If the hypothesis of a dynamical origin is correct, then the Hercules stream must be made of a mixture of stars of any population but mainly thin disk stars since they are more numerous. We believe that most of the previous studies of the thick disk may have included Hercules stars which may have perturbed the results. In this study we have carefully identified such stars. 
We have focused on the following key points: (1) the compilation of all the useful stellar parameters with only very accurate measurements; (2) the compilation of a large sample of disk stars with a significant fraction of thick disk stars; (3) the identification of pure thin disk and thick disk stars with a well-defined robust criterion. The stellar parameters useful for population studies and used in this paper are the velocities (distances, proper motions, radial velocities), metallicities $([\mathrm{Fe} / \mathrm{H}])$, abundance ratios and ages. The accuracy is guaranteed by selecting only stars within $100 \mathrm{pc}$ of the Sun (with a relative error of the Hipparcos parallax lower than 10\%) and recently submitted to detailed abundance studies from high resolution, high signal-to-noise spectra. In recent years, several groups have produced studies of elemental abundances involving a few dozen to hundreds of stars spanning a wide range of metallicity. We have combined them in order to build a large catalogue of stars with known elemental abundances. The kinematical classification of stars into the thin and thick disks is the most robust because it is not model-dependent and the kinematical properties of the two populations are well known. Moreover the combination of Hipparcos astrometric measurements and radial velocities from echelle spectrographs makes the $3 \mathrm{D}$ velocities of nearby stars very accurate. We have assigned stars to the thin disk, the thick disk and the Hercules stream on the basis of their $(U, V, W)$ velocity by computing their probability of belonging to the corresponding velocity ellipsoid.

We describe in Sect. 2.1 the construction of a large catalogue of elemental abundances from published data. Before combining the data we have analysed the agreement of abundances between authors. The distribution of abundances versus metallicity of the whole sample is shown. Section 3 describes the age determination and the kinematical data which was used to compute $(U, V, W)$ velocities and orbits. The kinematical classification performed to select three subsamples of stars representative of the thin disk, the thick disk and the Hercules stream is described in Sect. 4. In Sect. 5 we report our findings on the chemical properties of the three subsamples. Finally we discuss the age distributions, the lack of a vertical gradient in the thick disk and the case of several metal-rich outliers in Sect. 6 .

\section{The abundance catalogue}

\subsection{Construction of the catalogue}

In order to build a large sample of elemental abundances, we have compiled several studies from the literature presenting determinations of $\mathrm{O}, \mathrm{Na}, \mathrm{Mg}, \mathrm{Al}, \mathrm{Si}, \mathrm{Ca}, \mathrm{Ti}, \mathrm{Fe}, \mathrm{Ni}$ abundances. We have chosen in the literature the eleven most significant papers which present large lists of stars (Table 1).

Because all authors of spectral analyses do not use the same scales and methods, systematics may exist between their results which cannot be combined without a careful analysis and eventually some kind of homogenisation. As most of the eleven studies have stars in common, we were able to measure their agreement. We have observed that disagreements were more frequent in the metal-poor regime, and because we were
Table 1. List of datasets included in the catalogue with the number of stars in the chosen range of atmospheric parameters (see text) and the method used for the $T$ eff determination (ldr = line depth ratios): A04 $=$ Allende Prieto et al. (2004), B03 = Bensby et al. (2003), B04a = Bensby et al. (2004a), C00 = Chen et al. (2000), E93 = Edvardsson et al. (1993), F00 = Fulbright (2000), G03 = Gratton et al. (2003), M04 = Mishenina et al. (2004), N97 $=$ Nissen \& Schuster (1997), P00 = Prochaska et al. (2000), R03 = Reddy et al. (2003).

\begin{tabular}{|c|c|c|c|}
\hline Reference & $N$ & Elements & $T$ eff determination \\
\hline$\overline{\mathrm{A} 04}$ & 104 & $\mathrm{Fe}, \mathrm{Si}, \mathrm{Ca}, \mathrm{Mg}, \mathrm{Ti}, \mathrm{O}, \mathrm{Ni}$ & $b-y, B-V$ \\
\hline B03 & 66 & $\mathrm{Fe}, \mathrm{Si}, \mathrm{Ca}, \mathrm{Mg}, \mathrm{Ti}, \mathrm{Na}, \mathrm{Al}, \mathrm{Ni}$ & FeI \\
\hline B04a & 66 & $\mathrm{O}$ & FeI \\
\hline $\mathrm{COO}$ & 90 & $\mathrm{Fe}, \mathrm{Si}, \mathrm{Ca}, \mathrm{Mg}, \mathrm{Ti}, \mathrm{O}, \mathrm{Na}, \mathrm{Al}, \mathrm{Ni}$ & $b-y$ \\
\hline E93 & 188 & $\mathrm{Fe}, \mathrm{Si}, \mathrm{Ca}, \mathrm{Mg}, \mathrm{Ti}, \mathrm{O}, \mathrm{Na}, \mathrm{Al}, \mathrm{Ni}$ & $b-y$ \\
\hline F00 & 100 & $\mathrm{Fe}, \mathrm{Si}, \mathrm{Ca}, \mathrm{Mg}, \mathrm{Ti}, \mathrm{Na}, \mathrm{Al}, \mathrm{Ni}$ & $V-K, B-V, b-y$ \\
\hline G03 & 116 & $\mathrm{Fe}, \mathrm{Si}, \mathrm{Ca}, \mathrm{Mg}, \mathrm{Ti}, \mathrm{O}, \mathrm{Na}, \mathrm{Ni}$ & $B-V, b-y$ \\
\hline M04 & 174 & $\mathrm{Fe}, \mathrm{Si}, \mathrm{Mg}, \mathrm{Ni}$ & $\mathrm{H}_{\alpha}, \mathrm{ldr}$ \\
\hline N97 & 29 & $\mathrm{Fe}, \mathrm{Si}, \mathrm{Ca}, \mathrm{Mg}, \mathrm{Ti}, \mathrm{O}$ & FeI \\
\hline P00 & 10 & $\mathrm{Fe}, \mathrm{Si}, \mathrm{Ca}, \mathrm{Mg}, \mathrm{Ti}, \mathrm{O}, \mathrm{Na}, \mathrm{Al}, \mathrm{Ni}$ & FeI \\
\hline R03 & 181 & $\mathrm{Fe}, \mathrm{Si}, \mathrm{Ca}, \mathrm{Mg}, \mathrm{Ti}, \mathrm{Na}, \mathrm{Al}, \mathrm{Ni}$ & $b-y$ \\
\hline
\end{tabular}

interested in studying the disk population and specially the interface between the thin disk and the thick disk, we have eliminated all stars with $[\mathrm{Fe} / \mathrm{H}]<-1.3$. A larger dispersion observed among cold stars convinced us to build our sample with stars hotter than $4500 \mathrm{~K}$. The number of stars of the eleven studies which fall within these limits is given in Table 1.

The effective temperature is the most critical parameter in spectroscopic analyses and different methods for its determination may lead to inhomogeneous scales. A systematic difference in temperature translates into a systematic difference in metallicity thus in abundances relative to iron. The eleven studies considered for the catalogue have indeed used various methods, listed in Table 1. Our first concern before combining abundances from different authors was to quantify the agreement of their temperature scales. We have used as reference temperatures the determinations by Alonso et al. (1996), Blackwell \& Lynas-Gray (1998) and di Benedetto (1998), which are known to be in good agreement in the FGK regime. For each paper we have inspected the agreement of the temperature scale with the reference one. Table 2 gives the mean differences and dispersions obtained. The dispersions are reasonable, ranging from $68 \mathrm{~K}$ to $109 \mathrm{~K}$, typical of the accuracy of 50-100 K generally quoted in spectral analyses. The largest offsets are observed for N97 and P00, however they correspond to the samples with the poorest statistics. For the other studies, the offset is lower than $75 \mathrm{~K}$. Such a difference is not expected to strongly affect the abundances. According to M04, a difference of $100 \mathrm{~K}$ in $T$ eff translates into a difference of $0.07 \mathrm{dex}$ in $[\mathrm{Fe} / \mathrm{H}]$ and a lower value for other elements.

Most of the determinations of elemental abundances considered here are based on LTE spectral analyses. Two exceptions concern Na by G03 and Mg by M04. Rigourously, LTE and NLTE abundances should not be combined because they rely on different physics and may lead to systematic offsets depending on the spectral lines considered and the parameter 
Table 2. Statistics of the Teff comparison to Alonso et al. (1996), Blackwell \& Lynas-Gray (1998) and di Benedetto (1998).

\begin{tabular}{lrrr}
\hline \hline Ref. & $\Delta_{T \text { eff }}(\mathrm{K})$ & $\sigma_{T \text { eff }}(\mathrm{K})$ & $N$ \\
\hline A04 & 16 & 76 & 43 \\
B03 & -71 & 72 & 14 \\
C00 & 21 & 109 & 18 \\
E93 & -59 & 68 & 59 \\
F00 & 51 & 85 & 44 \\
G03 & -15 & 68 & 42 \\
M04 & -37 & 77 & 55 \\
N97 & -90 & 75 & 8 \\
P00 & 89 & 68 & 3 \\
R03 & 23 & 68 & 11 \\
All & -13 & 88 & 302 \\
\hline
\end{tabular}

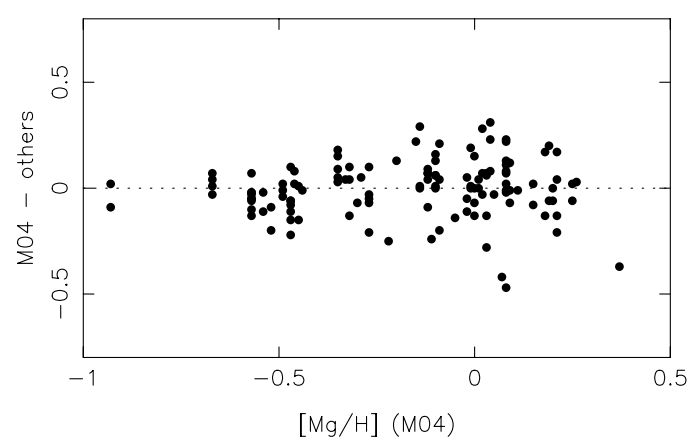

Fig. 1. Comparaison of $[\mathrm{Mg} / \mathrm{H}]$ computed under NLTE approximation by M04 and LTE $[\mathrm{Mg} / \mathrm{H}]$ computed by the other authors. No systematic trend can be observed. The mean difference is null, the dispersion 0.13 dex for 126 stars in common.

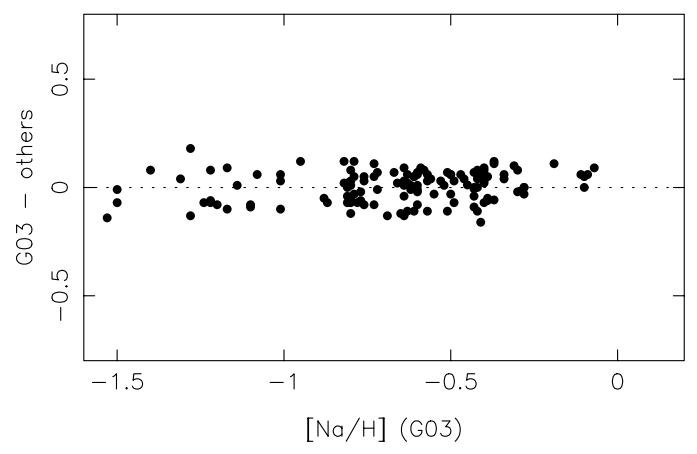

Fig. 2. Comparaison of $[\mathrm{Na} / \mathrm{H}]$ computed under NLTE approximation by $\mathrm{G} 03$ and LTE $[\mathrm{Na} / \mathrm{H}]$ computed by the other authors. No systematic trend can be observed. The mean difference is null, the dispersion 0.07 dex for 130 stars in common.

range of the stars. We have looked for such inconsistencies by comparing NLTE $[\mathrm{Mg} / \mathrm{H}]$ by M04 and NLTE $[\mathrm{Na} / \mathrm{H}]$ by $\mathrm{G} 03$ to their LTE couterparts by the other authors for stars in common (Figs. 1 and 2). The plots do not reveal systematic effects. For $\mathrm{Mg}$, the mean difference is null with a dispersion of $0.13 \mathrm{dex}$ for 126 stars in common. For $\mathrm{Na}$, the mean difference is also null with a dispersion 0.07 dex for 130 stars in common. The lack of an offset between these abundances led us to keep $\mathrm{Na}$

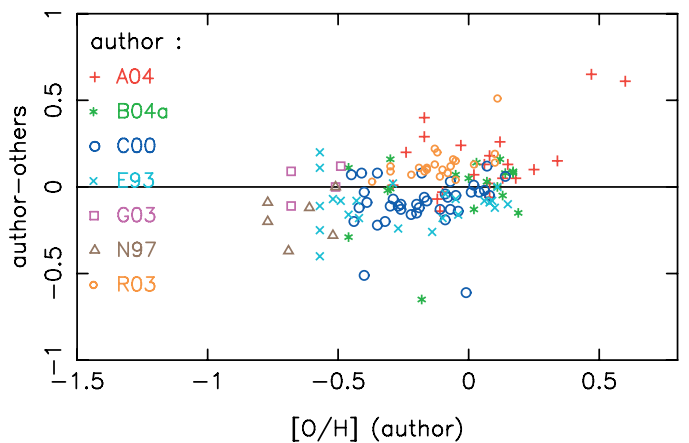

Fig. 3. Differences between $[\mathrm{O} / \mathrm{H}]$ on the LTE $6300 \AA$ scale derived by authors having stars in common.

and Mg NLTE determinations in the catalogue in order to enlarge our sample.

The case of oxygen is more complex. Oxygen abundances can be determined either from the forbidden [OI] lines at $6300 \AA$ and $6363 \AA$ or from the IR triplet OI lines at $7774 \AA$. The lines at $7774 \AA$ are strong and clean but affected by NLTE effects, whereas lines at $6300 \AA$ and $6363 \AA$ are weak and blended but unaffected by NLTE effects. According to the IAU recommendations, the $6300 \AA$ lines are the best indicators. We have thus selected in the considered papers only oxygen abundances on the LTE $6300 \AA$ scale, obtained either directly or with a correction. E93 have calibrated a correction to pass from the LTE oxygen abundances derived with the $7774 \AA$ line to those derived with the $6300 \AA$ line. C00 have used this transformation, and we have also used it to correct the LTE $7774 \AA$ determinations by N97 and P00. A04 and B04a directly used the $6300 \AA$ lines. G03 provided LTE $6300 \AA$ abundances for 22 stars and NLTE $7774 \AA$ determinations for 68 stars. We only kept the 22 LTE ones. Figure 3 shows the typical dispersion that affects oxygen abundances determined by different authors, even if they are on the same scale. The standard deviation of the plotted distribution is $0.19 \mathrm{dex}$ (132 values, $0.14 \mathrm{dex}$ when outliers are removed). There are 3 outliers: HD 172051 with $[\mathrm{O} / \mathrm{H}]=+0.47$ (A04) and $[\mathrm{O} / \mathrm{H}]=-0.18(\mathrm{~B} 04 \mathrm{a}), \mathrm{HD} 109303$ with $[\mathrm{O} / \mathrm{H}]=+0.11(\mathrm{R} 03)$ and $[\mathrm{O} / \mathrm{H}]=-0.40(\mathrm{C} 00)$, HD 210027 with $[\mathrm{O} / \mathrm{H}]=+0.60$ $(\mathrm{A} 04)$ and $[\mathrm{O} / \mathrm{H}]=-0.01(\mathrm{C} 00)$. A04 determinations are systematically higher than the others by $0.11 \mathrm{dex}$ and several stars have unexpectedly high values of [O/H] (HD 157214 and HD 144579 for instance). Considering that the total dispersion of differences between authors decreases to 0.13 dex (85 values) without A04, we have not considered the oxygen abundances by A04 in the final catalogue. It is beyond the scope of this paper to analyse why A04 does not seem to be on the same $[\mathrm{O} / \mathrm{H}]$ scale as the other authors.

We have continued our verification by comparing $[\mathrm{Fe} / \mathrm{H}]$ and abundance ratios of the different studies having at least 10 stars in common. We have observed the largest systematic differences, ranging from 0.08 to $0.10 \mathrm{dex}$, for $[\mathrm{Ti} / \mathrm{Fe}]$ and $[\mathrm{Mg} / \mathrm{Fe}](\mathrm{A} 04$ vs. E93), and for $[\mathrm{Mg} / \mathrm{Fe}]$ (A04 vs. C00). The median offset between the studies that could be compared is 0.02 . The largest dispersions concern $\mathrm{E} 93$ vs. $\mathrm{C} 00$ for $[\mathrm{Ti} / \mathrm{Fe}]$ and $[\mathrm{Al} / \mathrm{Fe}](0.10 \mathrm{dex})$, and M04 vs. A04 for $[\mathrm{Fe} / \mathrm{H}](0.11 \mathrm{dex})$. 
Table 3. For each element, number of stars with a determination of abundance.

\begin{tabular}{ccccccccc}
\hline \hline $\mathrm{Fe}$ & $\mathrm{O}$ & $\mathrm{Na}$ & $\mathrm{Mg}$ & $\mathrm{Al}$ & $\mathrm{Si}$ & $\mathrm{Ca}$ & $\mathrm{Ti}$ & $\mathrm{Ni}$ \\
\hline 743 & 415 & 568 & 725 & 509 & 743 & 641 & 630 & 739 \\
\hline
\end{tabular}

The median dispersion is 0.045 dex (a $3 \sigma$ rejection of a few outliers was performed to compute these statistics). These values are reasonable, considering realistic error bars 0.10 dex on $[\mathrm{Fe} / \mathrm{H}]$ and 0.06 dex on abundance ratios. We have thus constructed our catalogue of abundances by combining the results of the eleven selected papers, adopting a simple average when several determinations were available for a given star. The final catalogue consists of 743 stars, only available in electronic form at the CDS, Strasbourg. Table 3 gives the number of stars available per element.

\subsection{Abundance trends in the whole sample}

For each element X, Fig. 4 represents the distribution of the sample in the plane $[\mathrm{X} / \mathrm{Fe}]$ vs. $[\mathrm{Fe} / \mathrm{H}]$.

As expected, $\alpha$ elements ( $\mathrm{Mg}, \mathrm{Si}$, Ti and $\mathrm{Ca}$ ) show a similar behaviour with a decreasing trend as metallicity increases, the distribution being flat at solar metallicity. There are however some differences between these elements: $\mathrm{Mg}$ presents a higher dispersion at low metallicity, contrary to $\mathrm{Ca}$ which deacreases more regularly. Oxygen, which is also an $\alpha$ element continues to decrease in the metal rich regime and exhibits a large spread. The large dispersion of $[\mathrm{O} / \mathrm{Fe}]$ might be cosmic, but also due to measurement errors and inhomogeneities in the studies which were combined.

Interestingly, it seems that $\mathrm{Al}$ has the same behaviour as $\alpha$ elements, a similarity which has already been reported by E93 and B03. The two main features concerning $\mathrm{Na}$ are a change of dispersion at $[\mathrm{Fe} / \mathrm{H}] \simeq-0.70$, the metal-rich part showing a very low dispersion, and a rise at super-solar metallicities. The remarkable low dispersion of $[\mathrm{Ni} / \mathrm{Fe}]$ in the whole range of metallicity, measured to be 0.04 dex implies (1) that the different studies of our compilation are in excellent agreement for this element; (2) that the cosmic scatter is very low. As an iron peak element, $\mathrm{Ni}$ follows very well the metallicity. Most elements, except oxygen, show a rise of enhancement with respect to iron at $[\mathrm{Fe} / \mathrm{H}]>0$. Oxygen is the only element which is depleted in the metal rich regime. According to current models of chemical evolution of the Galaxy, $\alpha$ elements like $\mathrm{O}$ and $\mathrm{Mg}$ have the same source of production, hence should have the same trends. This is not observed in the metal rich regime but an interpretation of this falls outside the scope of this paper.

The Sun appears to be slightly deficient in all elements compared to stars with similar metallicity. The peculiarity of the solar abundance ratios was previously noticed by A04 and E93 with a tentative explaination related to its greater age.

\section{Velocities, orbits and ages}

In order to compute accurate velocities $(U, V, W)$ we first cross-correlated the abundance catalogue with Hipparcos
(ESA 1997), selecting stars with $\pi>10$ mas and $\frac{\sigma_{\pi}}{\pi}<0.10$. Then we searched for radial velocities in the following sources: Prugniel \& Soubiran (2001), Nidever et al. (2002), Nordström et al. (2004), Gratton et al. (2003), Barbier-Bossat et al. (2000). Positions, distances, proper motions and radial velocities have been combined to compute the 3 components $(U, V, W)$ of the spatial velocities with respect to the Sun. For most stars the uncertainty on the components of the spatial velocity is lower than $1 \mathrm{~km} \mathrm{~s}^{-1}$.

We were tempted to adopt the ages derived by Nordström et al. (2004) for 447 stars that we have in common with this catalogue. However their isochrone ages have been computed with photometric metallicities, of much lower accuracy than the spectroscopic ones that are available for our stars. Instead the derivation of ages was very kindly done for us by Frédéric Pont making use of the Bayesian method of Pont \& Eyer (2004) together with the high quality data (Hipparcos luminosities and spectroscopic temperatures and metallicities) that we have compiled and the theoretical isochrones from Padova (Girardi et al. 2000). Unfortunatly the current version of the software does not use $\alpha$-enhanced isochrones. On average the $\alpha$-enhancement of the most metal-poor stars of our sample is $[\alpha / \mathrm{Fe}]=+0.3$. Bensby et al. (2004b) have shown how $\alpha$-enhancement changes isochrone ages. In their example at $[\mathrm{Fe} / \mathrm{H}]=-0.70$ (see their Fig. 3), the shift between the isochrones at $[\alpha / \mathrm{Fe}]=+0.3$ and $[\alpha / \mathrm{Fe}]=0.0$ may reach $5 \mathrm{Gyr}$ in the evolved parts. Ages may be overestimated when the $\alpha$-enhancement is not taken into account. However it can be noticed in their Fig. 3 that the difference between enhanced and non-enhanced isochrones is equivalent at the first order to a temperature shift. As explained in Pont \& Eyer (2004), the temperature match between observations and models is one of the key points of the method. The temperature shift that is performed to make the model match the observations at a given metallicity also corrects the effects due to the neglected $[\alpha / \mathrm{Fe}]$ ratio. With the Bayesian method the most probable age is estimated together with a probability distribution function which was used to define a subsample of well-defined ages. Ages have been computed for all stars, but then we eliminated those corresponding to stars showing a wide or asymmetrical age probability distribution function, or with a position in the HR diagram far from the isochrones of same metallicity. With those restrictions we have good estimations of ages for 322 stars.

The orbital parameters have been computed by integrating the equations of motion in the galactic model of Allen \& Santillan (1993). When available we have adopted the age of the star as the integration time, otherwise a default value of 5 Gyr was adopted. The adopted velocity of the Sun with respect to the LSR is $(9.7,5.2,6.7) \mathrm{km} \mathrm{s}^{-1}$ (Bienaymé 1999, $\mathrm{U}$ positive towards the galactic center), the solar galactocentric distance $R_{\odot}=8.5 \mathrm{kpc}$ and circular velocity $V_{\mathrm{lsr}}=220 \mathrm{~km} \mathrm{~s}^{-1}$. In order to focus on the populations of the disk, we have eliminated from the sample several stars with halo kinematics. The final sample with abundances, velocities and orbits includes 639 stars.

Figures 5 and 6 present the dispersion of the vertical velocities $\mathrm{W}$ and the eccentricities of the orbits in several bins 

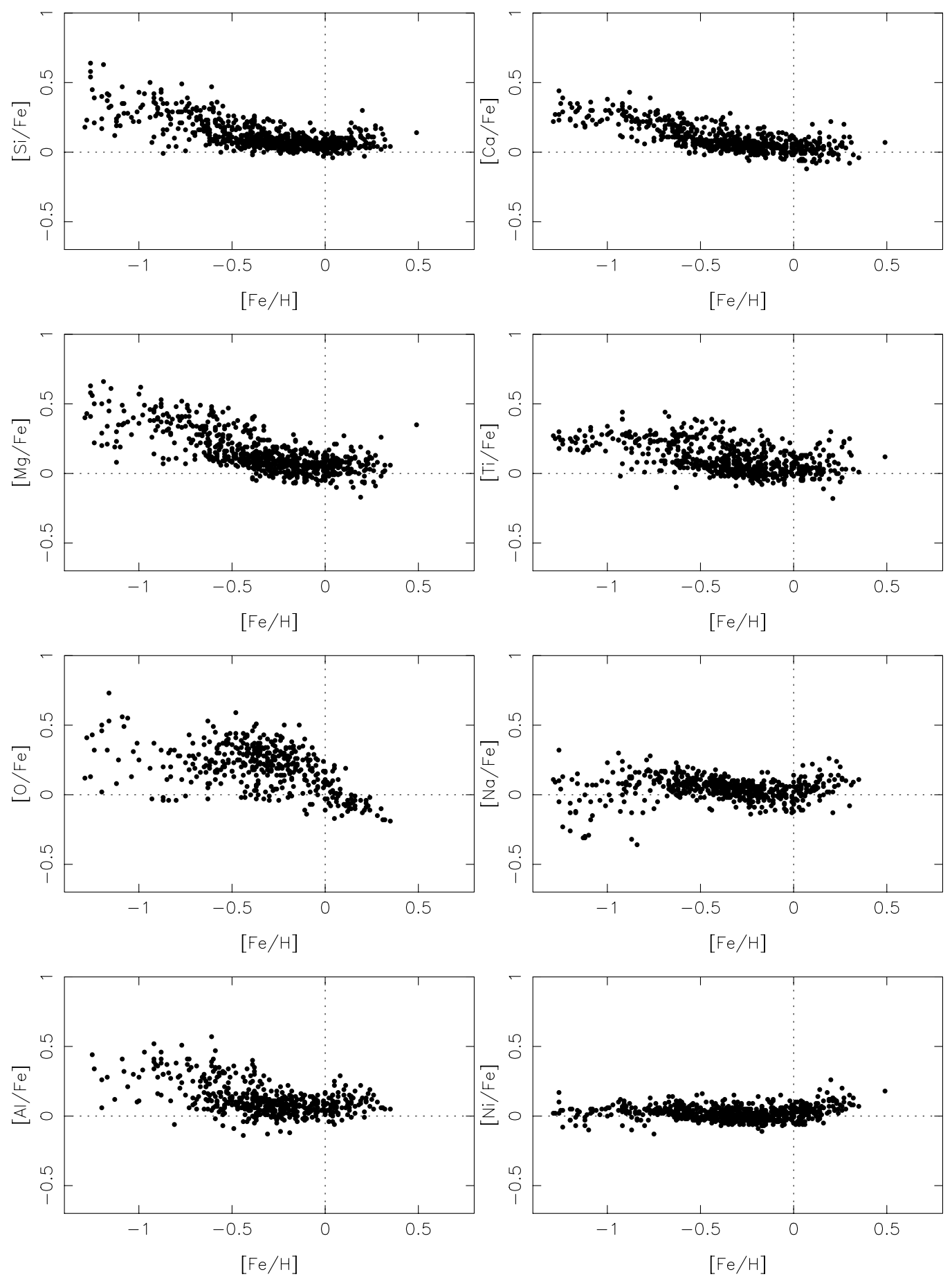

Fig. 4. $[\mathrm{X} / \mathrm{Fe}]$ vs. $[\mathrm{Fe} / \mathrm{H}]$.

of metallicity. Three regimes can be distinguished. The three bins of highest metallicity show a nearly flat distribution with $\sigma_{W} \simeq 15 \mathrm{~km} \mathrm{~s}^{-1}$ and $e c c \simeq 0.09$ showing that this metallicity interval is dominated by the thin disk. Interestingly the bin at $[\mathrm{Fe} / \mathrm{H}]=+0.26$ has $\sigma_{W}=20 \mathrm{~km} \mathrm{~s}^{-1}$ suggesting that there are super metal rich stars with hotter vertical kinematics. The increase of $\sigma_{W}$ and ecc as metallicity decreases in the next three bins reflects the mixture of the thin disk and the thick disk, with a growing number of thick disk stars. The last two bins of lowest metallicity saturate at $\sigma_{W} \simeq 45 \mathrm{~km} \mathrm{~s}^{-1}$ and $e c c \simeq$ 0.45. A smooth AMR is visible in Fig. 7. Several old super metal-rich stars are responsible of the jump and large dispersion in the last bin of metallicity. The same stars also have hot vertical kinematics.

\section{Kinematical classification}

In order to investigate the abundance trends in the thin disk and the thick disk separately, we have classified the stars into these populations, using the kinematical information. We have performed such a deconvolution in previous studies (Soubiran et al. 2003; and M04) where we have noticed that metal rich 


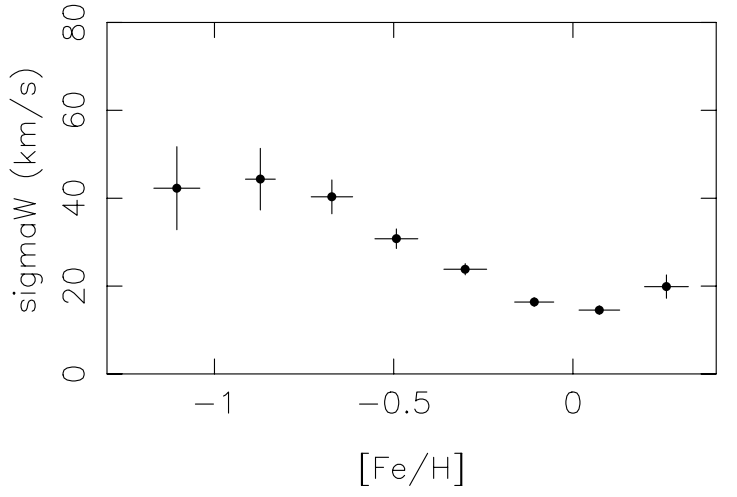

Fig. 5. $\sigma_{W}$ vs. $[\mathrm{Fe} / \mathrm{H}]$ of the whole sample in several bins of metallicity. Error bars correspond to the standard error for $\sigma_{W}\left(\sigma_{W} / \sqrt{2 N}\right)$ and standard deviation for $[\mathrm{Fe} / \mathrm{H}]$.

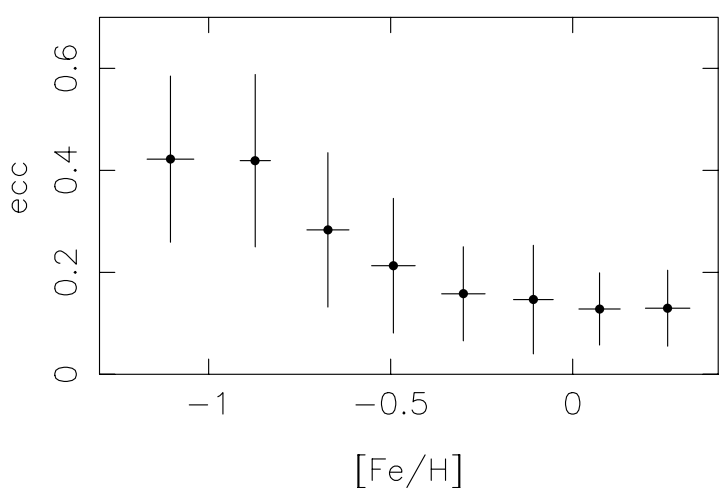

Fig. 6. Eccentricity of the orbits vs. $[\mathrm{Fe} / \mathrm{H}]$ of the whole sample in several bins of metallicity. Error bars correspond to the standard deviations.

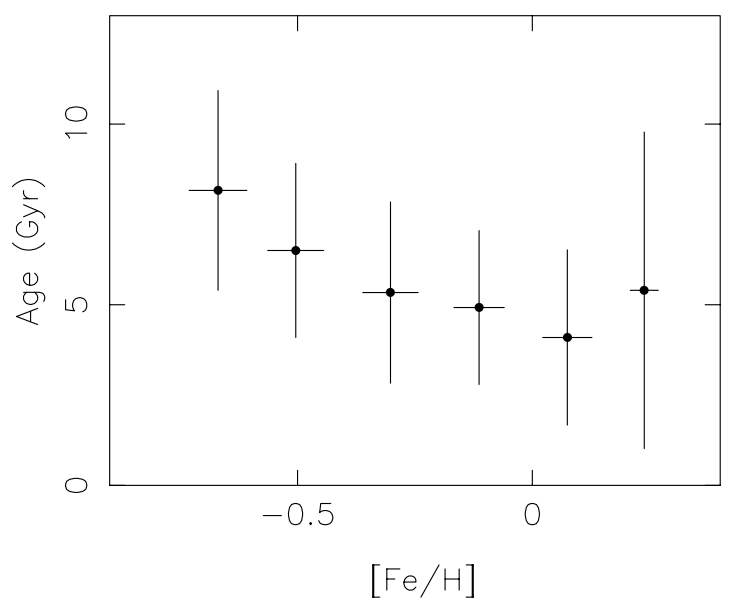

Fig. 7. Age vs. $[\mathrm{Fe} / \mathrm{H}]$ in several bins of metallicity for stars having well defined ages. Error bars correspond to the standard deviations.

stars with high eccentricity but low scale height were assigned to the thick disk whereas they could have another origin. The recent study by Famaey et al. (2004) has confirmed that $6.5 \%$ of the stars in the solar neigbourhood belong to the Hercules stream, which has a mean rotational motion with respect to the Sun very similar to that of the thick disk $\left(V=-51 \mathrm{~km} \mathrm{~s}^{-1}\right)$ with a significant radial motion of $U=-42 \mathrm{~km} \mathrm{~s}^{-1}$.
Table 4. Kinematical parameters of the three considered groups and their proportion in our sample.

\begin{tabular}{lrrr}
\hline \hline & Thin disk & Thick disk & Hercules \\
\hline$p(\%)$ & 72 & 19 & 9 \\
$U\left(\mathrm{~km} \mathrm{~s}^{-1}\right)$ & 0 & 0 & -42 \\
$V\left(\mathrm{~km} \mathrm{~s}^{-1}\right)$ & -12 & -51 & -51 \\
$W\left(\mathrm{~km} \mathrm{~s}^{-1}\right)$ & 0 & 0 & -7 \\
$\sigma_{U}\left(\mathrm{~km} \mathrm{~s}^{-1}\right)$ & 39 & 63 & 26 \\
$\sigma_{V}\left(\mathrm{~km} \mathrm{~s}^{-1}\right)$ & 20 & 39 & 9 \\
$\sigma_{W}\left(\mathrm{~km} \mathrm{~s}^{-1}\right)$ & 20 & 39 & 17 \\
$l_{v}(\mathrm{deg})$ & 0 & 0 & -5.7 \\
\hline
\end{tabular}

Famaey et al. (2004) have identified a total of six kinematical structures in their sample of nearby giants: the background stars corresponding to the mixed population of the thin disk, the high velocity group corresponding to the thick disk, the Hercules stream, the young group, the Hyades-Pleiades supercluster and the Sirius moving group. The three later groups correspond to young stars with kinematics typical of the thin disk. As can be seen in their Fig. 9, these groups of young stars appear as concentrations inside the velocity ellipsoid of the thin disk. They represent a peculiar class of thin disk stars and were not considered separately in this study. On the contrary the Hercules stream is of high interest for our probe of the thin disk to thick disk interface since its kinematics is exactly intermediate between to two populations. The authors explain that this stream has a dynamical origin. Contrary to a moving group which involves stars which were born at the same place and at the same time, a dynamical stream affects stars of any age and population. The effect of the bar in the central parts of the Galaxy is to move stars onto excentric orbits which tend then to mimic thick disk stars. Such stars, mainly thin disk stars with perturbed kinematics, could have polluted previous samples of thick disk stars selected on kinematical criteria and thus must be taken into account. The kinematical classification that we have performed is based on the assumption that our whole catalogue is dominated by three populations, the thin disk, the thick disk and the Hercules stream, all having gaussian velocity distributions.

We have computed for each star its probability of belonging to each of the three populations on the basis of its $(U, V, W)$ velocity and the Gaussian velocity ellispoid of the corresponding population (equations can be found in M04). The kinematical parameters (mean, standard deviation, vertex deviation) that define the velocity ellipsoids, as well as the proportions of the three populations in the sample, must be known. We adopt for the kinematical parameters those determined by Soubiran et al. (2003) for the old thin disk and the thick disk and by Famaey et al. (2004) for the Hercules stream (Table 4). The fraction of each population in the sample is more difficult to evaluate. We are aware that our whole sample might be biased in favour of high velocity and metal-poor stars due to the subject of the eleven studies we use. Consequently the proportion of thick disk and Hercules stars is expected to be higher than in the complete sample of Famaey et al. (2004) which is more representative of the content of the solar neighbourhood. In a similar way as described in M04 we have applied to our sample 


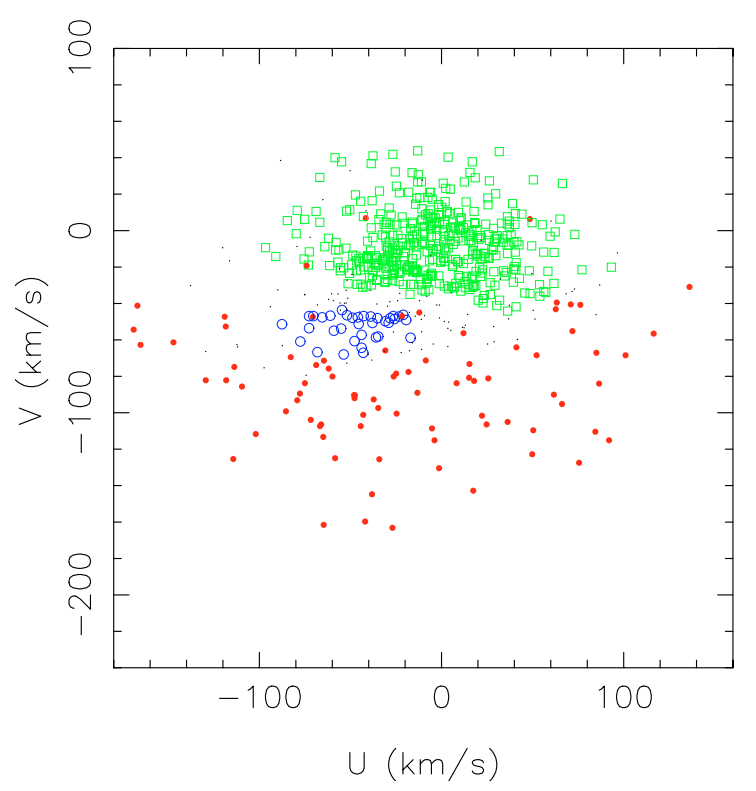

Fig. 8. The sample of 639 stars plotted in the $(U, V)$ plane. Thick disk stars are represented by red dots, thin disk stars by open green squares and Hercules stars by open blue circles. The small dots correspond to stars which could not be classified into the 3 subgroups.

of $(U, V, W)$ a non-informative algorithm of deconvolution of Gaussian distributions which showed that $72 \%$ of the stars have kinematics typical of the thin disk and $28 \%$ typical of the thick disk or the Hercules stream. Adopting the same ratio of thick disk to Hercules stream as Famaey et al. (2004), we have considered that $19 \%$ of our sample correspond to the thick disk, $9 \%$ to the Hercules stream.

According to the computed probabilities, we have selected three subsamples representative of the thin disk, the thick disk and the Hercules stream. The thin disk and the thick disk samples include respectively 428 and 84 stars having a probability higher than $80 \%$ of belonging to these populations. This probability cut was chosen as a compromise between having a sufficient number of thick disk stars and avoiding the contamination of the sample with stars with intermediate kinematics. It is more difficult to isolate Hercules stars since their velocity distribution greatly overlaps that of the thin and thick disks. Consequently there are only 5 stars with a probability higher than $80 \%$ of belonging to the Hercules stream. We had to lower the probability limit down to $50 \%$ to obtain 44 stars for the Hercules sample. Only 81 stars could not be classified into one of the three groups. These remaining stars represent a mixture of intermediate stars which are not considered in the following since our aim is to work on samples as pure as possible. The $(U$, $V$ ) plane and Toomre diagram of the whole sample is shown in Figs. 8 and 9, the three subsamples being highlighted in different colours and symbols. The Hercules stream is clearly visible as a concentration between the two disks.

How does our kinematical classification compare with those previously performed? A04, E93, F00, G03 and N97 have not considered the thick disk specifically. P00 selected 10 thick disk stars with $-1.1 \leq[\mathrm{Fe} / \mathrm{H}] \leq-0.4$ and $\mathrm{Zmax}>600 \mathrm{pc}$ and $-20 \leq V_{\mathrm{LSR}} \leq-100 \mathrm{~km} \mathrm{~s}^{-1}$. Only 5 of them remain in our

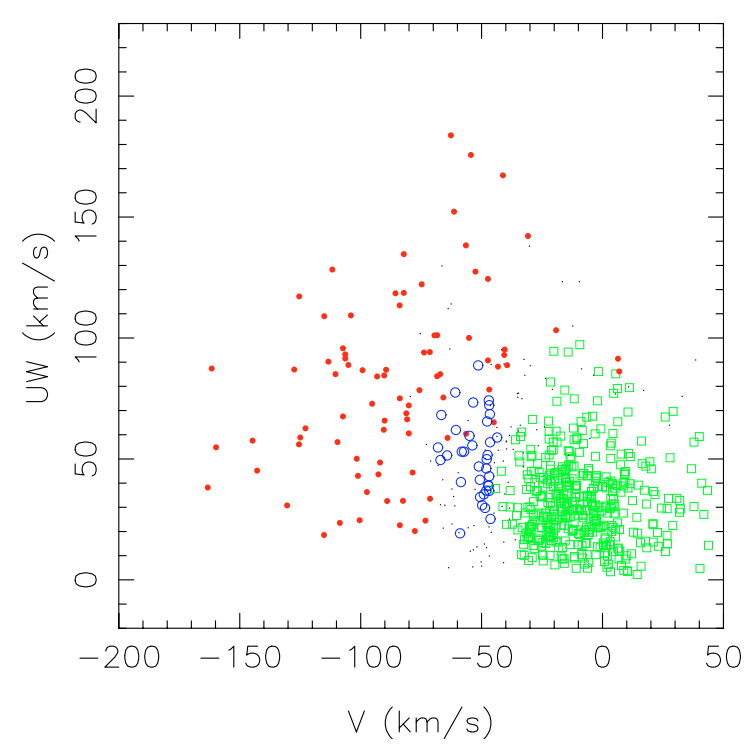

Fig. 9. As for Fig. 8, but in the Toomre diagram: $U W=$ $\sqrt{U^{2}+W^{2}} \mathrm{~km} \mathrm{~s}^{-1}$.

catalogue due to our restrictions on distances, all of them classified in the thick disk. If we apply P00's criteria to our catalogue, we only select 22 thick disk stars. These criteria are thus very efficient in avoiding intermediate or Hercules stars but they are far too restrictive to construct a large thick disk sample. C00 have proposed their stars with $[\mathrm{Fe} / \mathrm{H}] \leq-0.6$ and $V \leq-40 \mathrm{~km} \mathrm{~s}^{-1}$ to be thick disk stars. The same criterion applied to our catalogue leads to a mixed sample including 58 thick disk stars, 13 Hercules stars, 1 thin disk star and 12 intermediate stars. R03 have performed a more complicated selection of thick disk stars involving metallicity, mean of periand apogalactic distances $R_{\mathrm{m}}, V$ velocity and age. Doing the same without the age restriction (age $>10 \mathrm{Gyr}$ ), we select 25 thick disk stars and 4 Hercules stars. Taking the age restriction into account, only 3 thick disk stars are selected. These results show that a restriction on $Z$ max is more efficient than on $V$ or $R_{\mathrm{m}}$ to isolate pure thick disk stars. Nevertheless a restriction on $[\mathrm{Fe} / \mathrm{H}]$ is incompatible with the study of the thick disk's metallicity distribution and abundance trends. The kinematical classification performed by $\mathrm{B} 03$ is the only one that is quite similar to ours in its principle although they have used probability ratios. Among their 21 thick disk stars, only 2 of them (HD 210483 and HD 212231) are not part of our thick disk sample because of our high probability cut (they have a probability of $78 \%$ and $75 \%$ respectively of belonging to the thick disk with our classification). Among their 45 thin disk stars, HD 003735 has a high probability to belong to the Hercules stream $(73 \%)$ while HD 210277 is intermediate between the thin disk (probability 55\%) and the Hercules stream (probability $31 \%$ ). The classification by M04 is the same as ours, but without the Hercules stream. Among their 30 thick disk stars, 5 have moved into the Hercules stream and 5 are transition stars which could not be classified.

The catalogue including abundances, kinematical and orbital parameters, ages and membership probabilities is available at the CDS, in a single table. For each star it contains the 

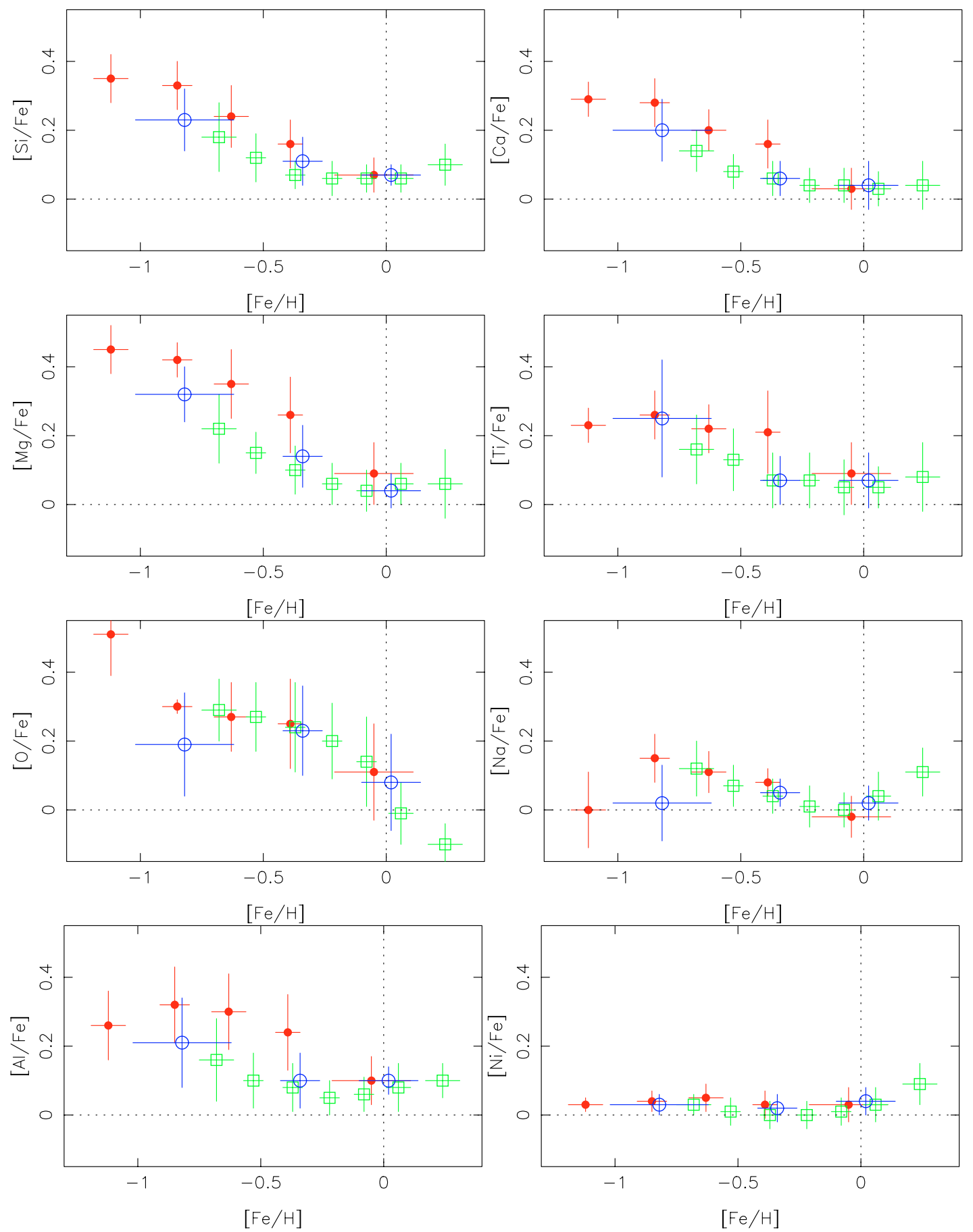

Fig. 10. Averaged $[\mathrm{X} / \mathrm{Fe}]$ vs. $[\mathrm{Fe} / \mathrm{H}]$ per bin of metallicity in the thin disk (green squares) in the thick disk (red filled circles) and in the Hercules stream (blue open circles). Errors bars correspond to the standard deviations around the mean value in each bin.

following information (when available): Col. 1 lists the name of the source, Col. 2 gives the metallicity [Fe/H], Cols. 3 to 10 give the abundances relative to iron of $\mathrm{O}, \mathrm{Na}, \mathrm{Mg}, \mathrm{Al}, \mathrm{Si}, \mathrm{Ca}$, $\mathrm{Ti}$ and $\mathrm{Ni}$, Cols. 11 to 13 give the three components $(U, V, W)$ of the heliocentric galactic velocity, Cols. 14 to 17 give the orbital parameters $R \min , R \max , Z \max$, eccentricity, Cols. 18 and 19 give the age and its relative error, Cols. 20 to 22 give the membership probability of the star to belong to the thin disk, the thick disk and the Hercules stream respectively. Another table, also available at the CDS, gives the parameters $T$ eff, $\log g$, $[\mathrm{Fe} / \mathrm{H}],[\alpha / \mathrm{Fe}]$ and $M \mathrm{v}$.

\section{Abundance trends}

The abundance trends for each kinematical group are shown in Fig. 10. The number of stars involved in this study has allowed us to bin the data in metallicity to highlight the abundance features of each population. Table 5 gives the mean abundance, standard deviation and number of stars in each metallicity bin. The observed features are the following:

- the thin disk and the thick disk overlap greatly in metallicity: there are stars with thin disk kinematics down to $[\mathrm{Fe} / \mathrm{H}]=-0.80$ and there are stars with thick disk 
Table 5. Mean abundance, standard deviation and number of stars in each metallicity bin.

\begin{tabular}{|c|c|c|c|c|c|c|c|c|c|}
\hline$\overline{\text { bin }}$ & 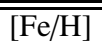 & {$[\mathrm{Si} / \mathrm{Fe}]$} & 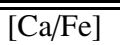 & {$[\overline{\mathrm{IMg} / \mathrm{Fe}]}$} & 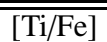 & $\overline{[\mathrm{OO} / \mathrm{Fe}]}$ & 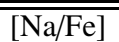 & $\overline{\overline{[\mathrm{Al} / \mathrm{Fe}]}}$ & $\overline{[\mathrm{Ni} / \mathrm{Fe}]}$ \\
\hline \multicolumn{10}{|c|}{ Thick disk } \\
\hline \multirow{3}{*}[\mathrm{Fe}/\mathrm{H}]{$<-1.00$} & -1.12 & 0.35 & 0.29 & 0.45 & 0.23 & 0.51 & 0.00 & 0.26 & 0.03 \\
\hline & 0.07 & 0.07 & 0.05 & 0.07 & 0.05 & 0.12 & 0.11 & 0.10 & 0.02 \\
\hline & 8 & 8 & 8 & 7 & 8 & 8 & 8 & 6 & 8 \\
\hline \multirow{3}{*}{$-1.00 \leq[\mathrm{Fe} / \mathrm{H}]<-0.75$} & -0.85 & 0.33 & 0.29 & 0.42 & 0.26 & 0.30 & 0.15 & 0.32 & 0.04 \\
\hline & 0.06 & 0.07 & 0.06 & 0.05 & 0.06 & 0.02 & 0.07 & 0.11 & 0.04 \\
\hline & 16 & 16 & 15 & 16 & 15 & 3 & 15 & 13 & 16 \\
\hline \multirow[t]{3}{*}{$-0.75 \leq[\mathrm{Fe} / \mathrm{H}]<-0.50$} & -0.63 & 0.24 & 0.20 & 0.35 & 0.23 & 0.27 & 0.11 & 0.30 & 0.05 \\
\hline & 0.07 & 0.09 & 0.06 & 0.10 & 0.07 & 0.10 & 0.06 & 0.11 & 0.04 \\
\hline & 31 & 31 & 28 & 31 & 28 & 13 & 28 & 27 & 31 \\
\hline \multirow[t]{3}{*}{$-0.50 \leq[\mathrm{Fe} / \mathrm{H}]<-0.25$} & -0.39 & 0.16 & 0.16 & 0.26 & 0.21 & 0.25 & 0.08 & 0.24 & 0.03 \\
\hline & 0.05 & 0.07 & 0.07 & 0.11 & 0.12 & 0.13 & 0.04 & 0.11 & 0.04 \\
\hline & 18 & 18 & 18 & 18 & 18 & 14 & 16 & 16 & 18 \\
\hline \multirow[t]{3}{*}{$-0.25 \leq[\mathrm{Fe} / \mathrm{H}]$} & -0.05 & 0.07 & 0.03 & 0.09 & 0.09 & 0.11 & -0.02 & 0.10 & 0.03 \\
\hline & 0.16 & 0.05 & 0.06 & 0.09 & 0.09 & 0.14 & 0.06 & 0.07 & 0.05 \\
\hline & 11 & 11 & 9 & 11 & 9 & 8 & 8 & 8 & 11 \\
\hline \multirow{4}{*}[\mathrm{Fe}/\mathrm{H}]{$<-0.60$} & & & & in disk & & & & & \\
\hline & -0.68 & 0.18 & 0.14 & 0.22 & 0.16 & 0.29 & 0.12 & 0.16 & 0.03 \\
\hline & 0.07 & 0.10 & 0.06 & 0.10 & 0.10 & 0.09 & 0.08 & 0.12 & 0.03 \\
\hline & 21 & 21 & 21 & 19 & 21 & 14 & 20 & 16 & 21 \\
\hline \multirow[t]{3}{*}{$-0.60 \leq[\mathrm{Fe} / \mathrm{H}]<-0.45$} & -0.53 & 0.12 & 0.08 & 0.15 & 0.13 & 0.27 & 0.07 & 0.10 & 0.01 \\
\hline & 0.04 & 0.07 & 0.05 & 0.06 & 0.09 & 0.10 & 0.06 & 0.08 & 0.04 \\
\hline & 37 & 37 & 35 & 36 & 33 & 23 & 33 & 33 & 37 \\
\hline \multirow[t]{3}{*}{$-0.45 \leq[\mathrm{Fe} / \mathrm{H}]<-0.30$} & -0.37 & 0.07 & 0.06 & 0.10 & 0.07 & 0.24 & 0.04 & 0.08 & 0.00 \\
\hline & 0.04 & 0.04 & 0.05 & 0.07 & 0.08 & 0.13 & 0.05 & 0.07 & 0.04 \\
\hline & 86 & 86 & 81 & 85 & 80 & 65 & 79 & 77 & 85 \\
\hline \multirow{3}{*}{$-0.30 \leq[\mathrm{Fe} / \mathrm{H}]<-0.15$} & -0.22 & 0.06 & 0.04 & 0.06 & 0.07 & 0.20 & 0.01 & 0.05 & 0.00 \\
\hline & 0.04 & 0.05 & 0.05 & 0.06 & 0.08 & 0.11 & 0.06 & 0.05 & 0.04 \\
\hline & 97 & 97 & 82 & 96 & 80 & 57 & 71 & 70 & 97 \\
\hline \multirow[t]{3}{*}{$-0.15 \leq[\mathrm{Fe} / \mathrm{H}]<0$} & -0.08 & 0.06 & 0.04 & 0.04 & 0.05 & 0.14 & 0.00 & 0.06 & 0.01 \\
\hline & 0.04 & 0.04 & 0.05 & 0.06 & 0.08 & 0.13 & 0.05 & 0.05 & 0.04 \\
\hline & 72 & 72 & 53 & 69 & 50 & 34 & 38 & 39 & 71 \\
\hline \multirow[t]{3}{*}{$0 \leq[\mathrm{Fe} / \mathrm{H}]<0.15$} & 0.06 & 0.06 & 0.03 & 0.06 & 0.05 & -0.01 & 0.04 & 0.08 & 0.03 \\
\hline & 0.05 & 0.04 & 0.05 & 0.06 & 0.06 & 0.09 & 0.07 & 0.07 & 0.05 \\
\hline & 83 & 83 & 65 & 81 & 65 & 38 & 53 & 49 & 82 \\
\hline \multirow[t]{3}{*}{$0.15 \leq[\mathrm{Fe} / \mathrm{H}]$} & 0.24 & 0.10 & 0.04 & 0.06 & 0.08 & -0.10 & 0.11 & 0.10 & 0.09 \\
\hline & 0.07 & 0.06 & 0.07 & 0.10 & 0.10 & 0.06 & 0.07 & 0.05 & 0.06 \\
\hline & 32 & 32 & 27 & 32 & 27 & 14 & 19 & 19 & 32 \\
\hline \multirow{4}{*}[\mathrm{Fe}/\mathrm{H}]{$<-0.50$} & & & Herc & les stream & & & & & \\
\hline & -0.82 & 0.23 & 0.20 & 0.32 & 0.25 & 0.19 & 0.02 & 0.21 & 0.03 \\
\hline & 0.20 & 0.09 & 0.09 & 0.08 & 0.17 & 0.15 & 0.11 & 0.13 & 0.03 \\
\hline & 8 & 8 & 8 & 8 & 8 & 4 & 6 & 3 & 8 \\
\hline \multirow[t]{3}{*}{$-0.50 \leq[\mathrm{Fe} / \mathrm{H}]<-0.20$} & -0.34 & 0.11 & 0.06 & 0.14 & 0.07 & 0.23 & 0.05 & 0.10 & 0.02 \\
\hline & 0.08 & 0.07 & 0.05 & 0.09 & 0.07 & 0.13 & 0.04 & 0.08 & 0.04 \\
\hline & 17 & 17 & 13 & 15 & 13 & 11 & 12 & 11 & 17 \\
\hline \multirow[t]{3}{*}{$-0.20 \leq[\mathrm{Fe} / \mathrm{H}]$} & 0.02 & 0.07 & 0.04 & 0.04 & 0.07 & 0.08 & 0.02 & 0.10 & 0.04 \\
\hline & 0.12 & 0.03 & 0.07 & 0.05 & 0.08 & 0.14 & 0.05 & 0.04 & 0.04 \\
\hline & 19 & 19 & 11 & 18 & 11 & 6 & 7 & 7 & 19 \\
\hline
\end{tabular}

kinematics at solar metallicity and above, with one of them in the super-metal-rich regime $([\mathrm{Fe} / \mathrm{H}]>+0.25)$;

- the $\alpha$ elements $(\mathrm{Mg}, \mathrm{Si}, \mathrm{Ti}$ and $\mathrm{Ca})$, as well as $\mathrm{Al}$ show the same behaviour: at solar metallicity the three populations blend; from $[\mathrm{Fe} / \mathrm{H}] \simeq-0.3$ to $[\mathrm{Fe} / \mathrm{H}] \simeq-0.7$, the thin and thick disks show parallel trends with an enhancement of these elements with respect to Fe in the thick disk;
- in the range $-0.70<[\mathrm{Fe} / \mathrm{H}]<-0.30,[\mathrm{Mg} / \mathrm{Fe}]$ and $[\mathrm{Al} / \mathrm{Fe}]$ of the thick disk exceed that of the thin disk by $\sim 0.15 \mathrm{dex}$;

- a change of slope (a "knee") is visible in the thick disk for $\mathrm{Si}$ and $\mathrm{Ca}$, less clearly for $\mathrm{Mg}$, at $[\mathrm{Fe} / \mathrm{H}] \simeq-0.7$;

- the only $\alpha$ element showing a knee in the thin disk is $\mathrm{O}$ at $[\mathrm{Fe} / \mathrm{H}] \simeq-0.5$, the decrease being steep above this value;

- for $\mathrm{O}$ the three populations blend but the most metal-poor bin exhibits a large enhancement of $[\mathrm{O} / \mathrm{H}]=+0.52$; 
- for $\mathrm{Na}$ and Ni the three populations blend;

- in the range $-0.60<[\mathrm{Fe} / \mathrm{H}]<-0.20,[\mathrm{Na} / \mathrm{Fe}]$ declines from $\sim+0.10$ dex to $\sim 0.00$ dex;

- super metal rich stars of the thin disk exhibit a pronounced overabundance of $\mathrm{Na}$ and $\mathrm{Ni}$;

- there are Hercules stars in the whole range of metallicity that exhibit abundance trends similar to those of the thin disk.

The overlapping metallicity distributions of the thin disk and the thick disk has been previously established (B03, M04). A question that is not yet answered is where the metallicity distribution of two disks stop. Concerning the metal-poor side of the thin disk, we find 47 stars with $[\mathrm{Fe} / \mathrm{H}]<-0.50$ among the 428 thin disk stars, the lowest value $([\mathrm{Fe} / \mathrm{H}]=-0.83)$ being reached by HD 134169. Our sample is biased and may exaggerate the fraction of metal-poor thin disk stars, but the existence of such stars is real and cannot be explained by measurement errors only. The super-metal-rich part of the thin disk seems to have a different chemical behaviour than the rest of the thin disk, the most obvious difference being observed for $\mathrm{Na}$ and $\mathrm{Ni}$. This raises the question of whether these stars have the same origin as the other thin disk stars. Looking in detail at their velocity shows that half of the thin disk stars with $[\mathrm{Fe} / \mathrm{H}]>+0.20$ have a motion consistent with that of the Hyades-Pleiades supercluster. Famaey et al. (2004) propose this stream as having originated from a common large molecular cloud, radially perturbed by a spiral wave. Our observation of similar high metallicities and peculiar abundance ratios for these stars is indeed in good agreement with the hypothesis of their formation from common material.

Concerning the thick disk, our data clearly prove the existence of metal-rich high velocity stars. However there are arguments in favour of and against the fact that the thick disk extends to solar metallicity and even beyond. M04 have discussed this issue and proposed that the thick disk stops at $[\mathrm{Fe} / \mathrm{H}] \simeq-0.30$ on the basis that more metal-rich stars may belong to the Hercules stream (whose velocity ellipsoid was not clearly defined at that time). Here we have carefully eliminated Hercules stars from the thick disk sample, but metalrich stars remain. Moreover, Feltzing (2004) claims to have observed metal-rich stars at a large distance above the plane where the thick disk dominates. If the thick disk really extends to solar metallicity then one has to explain why its $\alpha$ abundance trends are so different from the thin disk, except at solar metallicity. The case of these metal-rich high velocity stars is further discussed in the next section.

The conclusion about the overlapping metallicity distributions of the thin and thick disks is that $[\mathrm{Fe} / \mathrm{H}]$ is a very bad parameter by itself to identify the disk populations.

The $\alpha$ enhancement with respect to iron of the thick disk has been established by Gratton et al. (1996) and Fuhrmann (1998) but evidence of the decreasing parallel trends for the thin and thick disks is more recent and results from a careful kinematical deconvolution of the two disks by B03 and M04. The "knee", i.e. the change of slope from a constant enhancement to a decreasing one, is interpreted as the typical signature of SNIa to the enrichment of the interstellar gas from which the later thick disk stars formed (Feltzing et al. 2003). Our contribution to this issue is to quantify the enhancement as +0.14 dex in $\mathrm{Mg}$, a value larger than the measurement errors. The enhancement of the other $\alpha$ elements $\mathrm{Si}, \mathrm{Ca}$ and $\mathrm{Ti}$ is lower but their dispersion in the thick disk is also lower. The very good correlation of $\mathrm{Al}$ and $\mathrm{Mg}$ has been discussed by E93 from the point of view of yields of supernovae of different kinds. From the point of view of galactic structure and diagnosis to deconvolve the populations, $[\mathrm{Al} / \mathrm{Fe}]$ turns to be a very good parameter. We have tested the efficiency of the parameters $[\mathrm{Mg} / \mathrm{Fe}],[\alpha / \mathrm{Fe}]=0.25([\mathrm{Mg} / \mathrm{Fe}]+[\mathrm{Si} / \mathrm{Fe}]+[\mathrm{Ca} / \mathrm{Fe}]+[\mathrm{Ti} / \mathrm{Fe}])$ and $[\mathrm{Mg}+\mathrm{Al} / \mathrm{Fe}]=0.5([\mathrm{Mg} / \mathrm{Fe}]+[\mathrm{Al} / \mathrm{Fe}])$ by performing linear regressions with $[\mathrm{Fe} / \mathrm{H}]$. We obtain the following relations (restricted to $-0.80<[\mathrm{Fe} / \mathrm{H}]<-0.30$ ):

For the thin disk:

$$
\begin{aligned}
& {[\mathrm{Mg} / \mathrm{Fe}]=-0.37[\mathrm{Fe} / \mathrm{H}]-0.040, \sigma=0.067 \mathrm{dex}} \\
& {[\alpha / \mathrm{Fe}]=-0.29[\mathrm{Fe} / \mathrm{H}]-0.029, \sigma=0.052 \mathrm{dex}} \\
& {[\mathrm{Mg}+\mathrm{Al} / \mathrm{Fe}]=-0.29[\mathrm{Fe} / \mathrm{H}]-0.021, \sigma=0.061 \mathrm{dex}}
\end{aligned}
$$

For the thick disk:

$$
\begin{aligned}
& {[\mathrm{Mg} / \mathrm{Fe}]=-0.41[\mathrm{Fe} / \mathrm{H}]+0.097, \sigma=0.092 \mathrm{dex}} \\
& {[\alpha / \mathrm{Fe}]=-0.30[\mathrm{Fe} / \mathrm{H}]+0.071, \sigma=0.069 \mathrm{dex}} \\
& {[\mathrm{Mg}+\mathrm{Al} / \mathrm{Fe}]=-0.30[\mathrm{Fe} / \mathrm{H}]+0.128, \sigma=0.095 \text { dex. }}
\end{aligned}
$$

Comparing for each abundance ratio the offset $(\Delta)$ between the thin and thick disks with the total dispersion $\left(\sigma_{\mathrm{T}}\right)$, the most favourable ratio is $[\mathrm{Mg}+\mathrm{Al} / \mathrm{Fe}]$ with $\Delta=1.31 \sigma_{\mathrm{T}}(\Delta=$ $\left.0.148 \mathrm{dex}, \sigma_{\mathrm{T}}=0.113 \mathrm{dex}\right)$, whereas $\Delta=1.20 \sigma_{\mathrm{T}}(\Delta=$ $\left.0.137 \mathrm{dex}, \sigma_{\mathrm{T}}=0.114 \mathrm{dex}\right)$ is obtained for $[\mathrm{Mg} / \mathrm{Fe}]$ and $\Delta=1.16 \sigma_{\mathrm{T}}\left(\Delta=0.100 \mathrm{dex}, \sigma_{\mathrm{T}}=0.086 \mathrm{dex}\right)$ for $[\alpha / \mathrm{Fe}]$.

If there is a jump in the enhancement of oxygen at $[\mathrm{Fe} / \mathrm{H}]<$ -1.0 , mainly due to the contribution of G03, our data do not show any enhancement of $[\mathrm{O} / \mathrm{Fe}]$ in the thick disk similar to the other $\alpha$ elements, a feature that was observed by B04a. As mentioned previously, the dispersions are large and may reflect the greater uncertainties that affect $\mathrm{O}$ abundance determinations compared to other elements. There is however a clear knee at $[\mathrm{Fe} / \mathrm{H}]=-0.5$, the decrease of $[\mathrm{O} / \mathrm{Fe}]$ being steeper at larger metallicites, which suggests an abrupt change in the relative role of SN I and SN II.

The fact that the Hercules stream occupies a wide range of metallicity with abundance trends similar to those of the thin disk is in good agreement with the dynamical hypothesis described by Famaey et al. (2004) since the perturbation of a rotating bar is supposed to affect non-contemporary but essentially thin disk stars.

\section{AMR, vertical gradient, metal-rich stars}

Figures 11 and 12 show that the thick disk is older than the thin disk in the whole range of metallicity. Thick disk stars range from 7 to 13 Gyr with an average of $9.6 \pm 0.3$ Gyr (median $9.5 \mathrm{Gyr}$ ). Several thin disk stars are found with ages greater than 10 Gyr, for instance HD 127334 and HD 190248, which are also metal-rich and appear as outliers. An AMR is visible in the thin disk, in the sense the most metal-poor thin disk stars are 


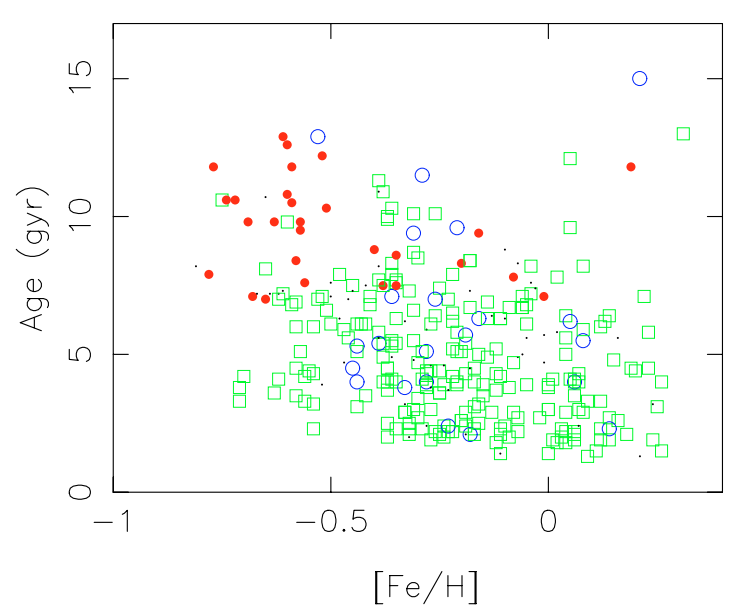

Fig. 11. Age vs. $[\mathrm{Fe} / \mathrm{H}]$ for stars having well-defined ages. As in Figs. 8 and 9, the different symbols indicate thin disk, thick disk and Hercules stars.

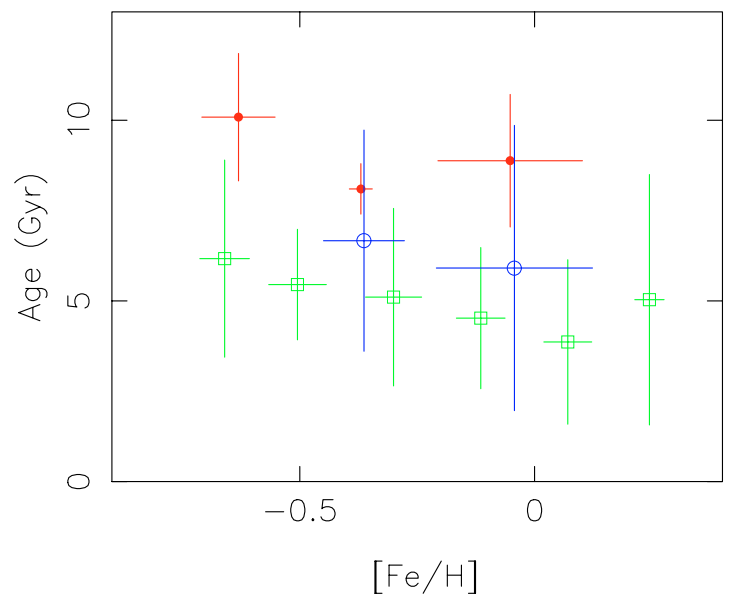

Fig. 12. Age vs. $[\mathrm{Fe} / \mathrm{H}]$ for stars having well-defined ages. As in Figs. 8 and 9, the different symbols indicate thin disk, thick disk and Hercules stars.

older than those with solar metallicity by $2.3 \mathrm{Gyr}$ on average. The AMR in the thick disk is more difficult to establish: the statistics are poor, only 29 stars having an age determination. It depends also whether one classifies the metal-rich stars into the thick disk or not. Bensby et al. (2004b) observed an AMR in the thick disk from a larger kinematically selected sample, but with ages determined from photometric metallicities. They obtain median ages of $13.0 \mathrm{Gyr}$ at $[\mathrm{Fe} / \mathrm{H}]=-0.60$ and $11.2 \mathrm{Gyr}$ at $[\mathrm{Fe} / \mathrm{H}]=-0.40$. Our age scale is lower but we obtain a similar difference of age in the same metallicity bins (10.1 Gyr and 8.1 Gyr). The metal-rich bin of the thick disk does not follow the same trend. If one considers this bin to be part of the thick disk, then the AMR hypothesis is not valid any longer. However we have several arguments against the metal-rich high velocity stars being part of the thick disk (see below) and thus we interpret our data as consistent with the existence of an AMR in the thick disk. Bensby et al. (2004b) have estimated that star formation was active in the thick disk for $5 \mathrm{Gyr}$, but this result is based on a wide metallicity distribution up to $[\mathrm{Fe} / \mathrm{H}]=0$. Our results favour a shorter timescale for star formation, of the order of 2-3 Gyr.

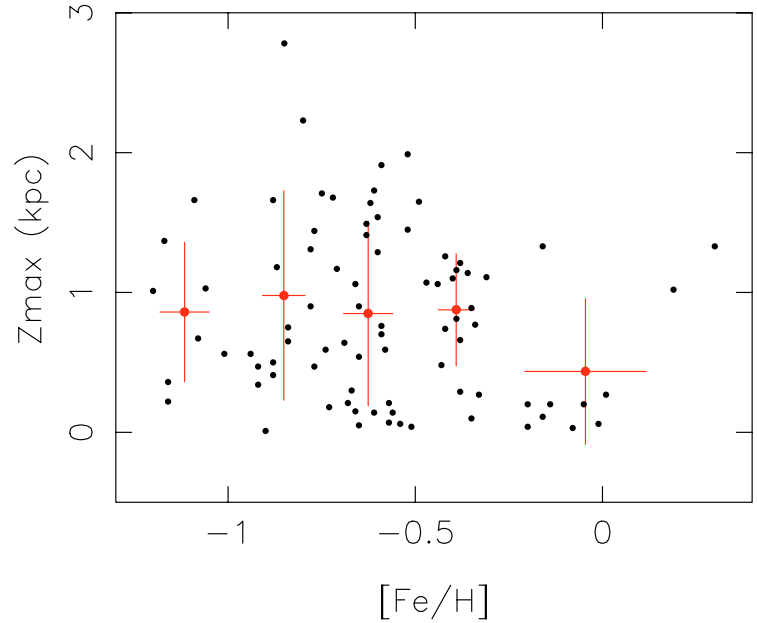

Fig. 13. Zmax, the maximal distance to the plane reached by the orbit, in several bins of metallicity for thick disk stars.

Our data are also compatible with a hiatus in star formation between the formation of the thick disk and the thin disk. In the range $-0.80<[\mathrm{Fe} / \mathrm{H}]<-0.30$, the mean age of the two disks differs by 4 Gyr. The reality of this offset is related to the scatter of the age distribution in each population. The comparison with the Hercules stream is very interesting in that sense. According to Fig. 11, the Hercules stream is made of stars of all ages. Consequently the age dispersion in its metallicity bins is very large. On the contrary, the thin and thick disks show much lower dispersions. The age dispersion in each bin results from the convolution of the cosmic scatter and the measurement errors of individual ages. We are aware that the uncertainty on ages is large despite the care we took to construct a clean sample. The median of the estimated errors is 2.7 Gyr. This means that the cosmic scatter of ages in each metallicity bin is quite low and the age offset between the two disks significant, pointing to a possible interruption of star formation at the end of the thick disk formation and the beginning of the thin disk formation. The large age dispersion of the Hercules stream favours the dynamical hypothesis proposed by Famaey et al. (2004) as its origin.

The existence of a vertical gradient of metallicity in the thick disk has important consequences for the choice of the most probable scenario of its formation. The relation between the maximal height above the plane, $Z$ max, and the metallicity is shown in Fig. 13. As previously mentioned in Soubiran et al. (2003) and M04, a transition occurs at $[\mathrm{Fe} / \mathrm{H}]=-0.3$. For stars more metal-poor than this value, the relation $\mathrm{Zmax}$ vs. $[\mathrm{Fe} / \mathrm{H}]$ is flat, consistent with no vertical gradient.

The question of whether the stars assigned to the thick disk in the highest metallicity bin are real thick disk stars is important to clarify the extent of the metallicity distribution of the thick disk and the existence of an AMR. It can be seen that thick disk stars with $[\mathrm{Fe} / \mathrm{H}]>-0.3$ are of two kinds: 8 stars have a flat distribution $(Z \max <300 \mathrm{pc})$ which is unlikely for thick disk stars, whereas 3 stars appear as outliers with Zmax $>1 \mathrm{kpc}$. The 8 stars come from A04, B03, M04 and R03. Therefore their distribution is not due to a peculiar selection bias of one of the eleven papers used to construct our sample. 
The 3 outliers have also been studied by several authors: HD 003628 by B03, F00 and G03, HD 145148 by B03 and HD 190360 by B03 and M04. The combination of the eleven papers used to construct the catalogue of abundance did not fill the gap of thick disk stars having $[\mathrm{Fe} / \mathrm{H}]>0$ and $300 \mathrm{pc}<Z \max <1 \mathrm{kpc}$. Finally stars with $[\mathrm{Fe} / \mathrm{H}]>-0.3$ assigned to the thick disk differ from the rest of the thick disk in three points: their vertical distribution is inhomogeneous, their $\alpha$ abundances are similar to that of the thin disk and they do not follow the AMR of the thick disk. Their nature has still to be clarified with a complete sample.

\section{Summary}

We have compiled a large catalogue of high quality stellar parameters of FGK stars to probe the properties of the thin disk and the thick disk in the metallicity interval $-1.30<[\mathrm{Fe} / \mathrm{H}]<+0.50$. This catalogue includes 743 stars with abundance ratios of several $\alpha$ and iron peak elements, 639 stars with abundance ratios and accurate $(U, V, W)$ velocities and orbits and 322 stars with abundance ratios, kinematical data and age estimations. The data are available in electronic tables at the CDS. We have used these data to investigate the metallicity, abundance ratios and age distributions of three kinematical groups with well-defined velocity ellipsoids. Three subsamples have been selected on the basis of $(U, V, W)$ velocities to be representative of the thin disk (428 stars), of the thick disk (84 stars) and the Hercules stream (44 stars).

Our results confirm previous well established findings:

- The thin disk and the thick disk overlap in metallicity and exhibit parallel slopes of $[\alpha / \mathrm{Fe}]$ vs. $[\mathrm{Fe} / \mathrm{H}]$ in the range $-0.80<[\mathrm{Fe} / \mathrm{H}]<-0.30$, the thick disk being enhanced.

- The thick disk is older than the thin disk.

We bring new constraints on more controversial issues:

- The thin disk extends down to $[\mathrm{Fe} / \mathrm{H}]=-0.80$ and exhibits low dispersions in its abundance trends.

- The thick disk also shows smooth abundance trends with low dispersions. The change of slope, which reflects the contribution of the different supernovae to the ISM enrichment, is visible in $[\mathrm{Si} / \mathrm{Fe}]$ vs. $[\mathrm{Fe} / \mathrm{H}]$ and $[\mathrm{Ca} / \mathrm{Fe}]$ vs. $[\mathrm{Fe} / \mathrm{H}]$ at $[\mathrm{Fe} / \mathrm{H}] \simeq-0.70$, less clearly in $[\mathrm{Mg} / \mathrm{Fe}]$ vs. $[\mathrm{Fe} / \mathrm{H}]$.

- An AMR is visible in the thin disk, the most metal-poor stars having 6.2 Gyr on average, those with solar metallicity 3.9 Gyr.

- Ages in the thick disk range from 7 to 13 Gyr with an average of $9.6 \pm 0.3$ Gyr. There is tentative evidence of an AMR extending over 2-3 Gyr.

- We do not find any evidence of a vertical metallicity gradient in the thick disk.

- $[\mathrm{O} / \mathrm{Fe}]$ decreases over the metallicity range with a change of slope at $[\mathrm{Fe} / \mathrm{H}]=-0.50$.

- The most metal-rich stars assigned to the thin disk do not follow its global trends. They are significantly enhanced in all elements (particularly in $\mathrm{Na}$ and $\mathrm{Ni}$ ) except in $\mathrm{O}$ which is clearly depleted. They also have a larger dispersion in age. Half of these stars are probable members of the HyadesPleiades supercluster, two others are surprisingly old.
We have also obtained new results:

- The slope of $[\alpha / \mathrm{Fe}]$ vs. $[\mathrm{Fe} / \mathrm{H}]$ in the interval $-0.80<[\mathrm{Fe} / \mathrm{H}]<-0.30$ has been estimated for the two disks. The enhancement of $\alpha$ elements in the thick disk has been quantified to be +0.10 dex. $[\mathrm{Mg} / \mathrm{Fe}]$ is more efficient than $[\alpha / \mathrm{Fe}]$ in separating the two disks with an offset of +0.14 dex. Combining $[\mathrm{Al} / \mathrm{Fe}]$ and $[\mathrm{Mg} / \mathrm{Fe}]$ is even better, with an offset of +0.15 dex between the two disks and a lower dispersion.

- The age difference between the thin and thick disks has been quantified to be $4 \mathrm{Gyr}$, suggesting an interruption in star formation between their formation.

- The Hercules stream is found to span the whole metallicity and age range. Its chemical properties are similar to those of the thin disk. This favours the dynamical hypothesis for its origin, related to the influence of the central bar of the Galaxy which affects non-contemporary stars.

- Metal-rich stars assigned to the thick disk do not follow its global trends. The nature of these stars remains to be clarified.

In this paper we have focused on observational results. The next step is to compare them to predictions of models. We believe that this dataset and our findings bring new strong constraints to models of formation of the two components of the galactic disk, and to models of chemical evolution.

Acknowledgements. We warmly thank Frédéric Pont who kindly computed the ages with his Bayesian method. This research has made use of the SIMBAD and VIZIER databases, operated at CDS, Strasbourg, France. It has used data from the ESA Hipparcos satellite (Hipparcos and Tycho-2 catalogues).

\section{References}

Allen, C., \& Santillan, A. 1993, RMxAA, 25, 39

Allende Prieto, C., Barklem, P. S., Lambert, D. L., \& Cunha, K. 2004, A\&A, 420, 183 (A04)

Alonso A., Arribas S., \& Martinez-Roger C. 1996, A\&AS, 117, 227

Barbier-Brossat, M., \& Figon, P. 2000 A\&AS, 142, 217

Bensby, T., Feltzing, S., \& Lundström, I. 2003, A\&A, 410, 527 (B03)

Bensby, T., Feltzing, S., \& Lundström, I. 2004a, A\&A, 415, 155 (B04a)

Bensby, T., Feltzing, S., \& Lundström, I. 2004b, A\&A, 421, 969

Blackwell, D. E., \& Lynas-Gray, A. E. 1998, A\&AS, 129, 505

Bienaymé, O. 1999 A\&A, 341, 86

Chen, Y. Q., Nissen, P. E., Zhao, G., Zhang, H. W., \& Benoni, T. 2000, A\&AS, 141, 491 (C00)

di Benedetto, G. P. 1998, A\&A, 339, 858

Edvardsson, B., Andersen, J., Gustafsson, B., et al. 1993, A\&A, 275, 101 (E93)

ESA 1997, The HIPPARCOS and TYCHO catalogues Noordwijk, Netherlands: ESA Publ. Division

Famaey, B., Jorissen, A., Luri, X., et al. 2005, A\&A, 430, 165

Feltzing, S., Bensby, T., \& Lundström, I. 2003, A\&A, 397, L1

Feltzing, S. 2004, Chemical Abundances and Mixing in Stars in the Milky Way Galaxy and its Satellites, ESO Astrophysics Symposia, ed. L. Pasquini \& S. Randich (Springer-Verlag Press) [arXiv:astro-ph/0411172] 
Fuhrmann, K. 1998, A\&A, 338, 161

Fulbright, J. P. 2000, AJ, 120, 1841 (F00)

Girardi, L., Bressan, A., Bertelli, G., \& Chiosi, C. 2000, A\&AS, 141, 371

Gratton, R. G., Carretta, Claudi, R., Lucatello, S., \& Barbieri, M. 2003, A\&A, 404, 187 (G03)

Gratton, R. G., Carretta, E., Mateucci, F., \& Snenden, C. 1996, in Formation of the Galactic Halo...Inside and Out, ed. H. Morisson, \& A. Sarajedini, ASP Conf. Ser., 92, 307

Mishenina, T. V., Soubiran, C., Kovtyukh, V.V., \& Korotin, S.A. 2004, A\&A, 418, 551 (M04)
Nidever, D. L., Marcy, G. W., Butler, R. P., Fischer, D. A., \& Vogt, S. S. 2002, ApJS, 141, 503

Nissen, P. E., \& Schuster, W. J. 1997, A\&A, 326, 751 (N97)

Nordström, B., Mayor, M., Andersen, J., et al. 2004, A\&A, 418, 989

Pont, F., \& Eyer, L. 2004, MNRAS, 351, 487

Prochaska, J. X., Naumov, S. O., Carney, B. W., McWilliam, A., \& Wolfe, A. M. 2000, ApJ, 120, 2513 (P00)

Prugniel, P., \& Soubiran, C. 2001, A\&A, 369, 1048

Reddy, B. E., Tomkin, J., Lambert, D. L., \& Allende Prieto, C. 2003, MNRAS, 340, 304 (R03)

Soubiran, C., Bienaymé, O., \& Siebert, A. 2003, A\&A, 398, 141 